\title{
Cartesian Products of Family of Real Linear Spaces
}

\author{
Hiroyuki Okazaki \\ Shinshu University \\ Nagano, Japan
}

Summary. In this article we introduced the isomorphism mapping between cartesian products of family of linear spaces [4]. Those products had been formalized by two different ways, i.e., the way using the functor [:X,Y:] and ones using the functor "product". By the same way, the isomorphism mapping was defined between Cartesian products of family of linear normed spaces also.

MML identifier: $\underline{\text { PRVECT_3}}$, version: $\underline{7.11 .07} 4.156 .1112$

The notation and terminology used in this paper are introduced in the following articles: [5], [1], [16], [11], [3], [6], [17], [7], [8], [15], [14], [2], [13], [12], [20], [18], [10], [19], and [9].

\section{Preliminaries}

One can prove the following propositions:

(1) Let $D, E, F, G$ be non empty sets. Then there exists a function $I$ from $D \times E \times(F \times G)$ into $D \times F \times(E \times G)$ such that

(i) $I$ is one-to-one and onto, and

(ii) for all sets $d, e, f, g$ such that $d \in D$ and $e \in E$ and $f \in F$ and $g \in G$ holds $I(\langle d, e\rangle,\langle f, g\rangle)=\langle\langle d, f\rangle,\langle e, g\rangle\rangle$. 
(2) Let $X$ be a non empty set and $D$ be a function. Suppose $\operatorname{dom} D=\{1\}$ and $D(1)=X$. Then there exists a function $I$ from $X$ into $\prod D$ such that $I$ is one-to-one and onto and for every set $x$ such that $x \in X$ holds $I(x)=\langle x\rangle$.

(3) Let $X, Y$ be non empty sets and $D$ be a function. Suppose $\operatorname{dom} D=$ $\{1,2\}$ and $D(1)=X$ and $D(2)=Y$. Then there exists a function $I$ from $X \times Y$ into $\prod D$ such that $I$ is one-to-one and onto and for all sets $x, y$ such that $x \in X$ and $y \in Y$ holds $I(x, y)=\langle x, y\rangle$.

(4) Let $X$ be a non empty set. Then there exists a function $I$ from $X$ into $\Pi\langle X\rangle$ such that $I$ is one-to-one and onto and for every set $x$ such that $x \in X$ holds $I(x)=\langle x\rangle$.

Let $X, Y$ be non-empty non empty finite sequences. Observe that $X^{\frown} Y$ is non-empty.

We now state two propositions:

(5) Let $X, Y$ be non empty sets. Then there exists a function $I$ from $X \times Y$ into $\Pi\langle X, Y\rangle$ such that $I$ is one-to-one and onto and for all sets $x, y$ such that $x \in X$ and $y \in Y$ holds $I(x, y)=\langle x, y\rangle$.

(6) Let $X, Y$ be non-empty non empty finite sequences. Then there exists a function $I$ from $\prod X \times \prod Y$ into $\prod\left(X^{\frown} Y\right)$ such that $I$ is one-to-one and onto and for all finite sequences $x, y$ such that $x \in \prod X$ and $y \in \prod Y$ holds $I(x, y)=x^{\frown} y$.

Let $G, F$ be non empty additive loop structures. The functor prodadd $(G, F)$ yielding a binary operation on (the carrier of $G) \times($ the carrier of $F$ ) is defined by:

(Def. 1) For all points $g_{1}, g_{2}$ of $G$ and for all points $f_{1}, f_{2}$ of $F$ holds $(\operatorname{prodadd}(G, F))\left(\left\langle g_{1}, f_{1}\right\rangle,\left\langle g_{2}, f_{2}\right\rangle\right)=\left\langle g_{1}+g_{2}, f_{1}+f_{2}\right\rangle$.

Let $G, F$ be non empty RLS structures. The functor prodmlt $(G, F)$ yielding a function from $\mathbb{R} \times(($ the carrier of $G) \times($ the carrier of $F)$ ) into (the carrier of $G) \times($ the carrier of $F)$ is defined by:

(Def. 2) For every element $r$ of $\mathbb{R}$ and for every point $g$ of $G$ and for every point $f$ of $F$ holds $(\operatorname{prodmlt}(G, F))(r,\langle g, f\rangle)=\langle r \cdot g, r \cdot f\rangle$.

Let $G, F$ be non empty additive loop structures. The functor prodzero $(G, F)$ yields an element of (the carrier of $G) \times($ the carrier of $F$ ) and is defined by:

(Def. 3) prodzero $(G, F)=\left\langle 0_{G}, 0_{F}\right\rangle$.

Let $G, F$ be non empty additive loop structures. The functor $G \times F$ yielding a strict non empty additive loop structure is defined by:

(Def. 4) $G \times F=\langle($ the carrier of $G) \times($ the carrier of $F)$, $\operatorname{prodadd}(G, F)$, $\operatorname{prodzero}(G, F)\rangle$.

Let $G, F$ be Abelian non empty additive loop structures. Observe that $G \times$ $F$ is Abelian. 
Let $G, F$ be add-associative non empty additive loop structures. Note that $G \times F$ is add-associative.

Let $G, F$ be right zeroed non empty additive loop structures. Note that $G \times$ $F$ is right zeroed.

Let $G, F$ be right complementable non empty additive loop structures. Note that $G \times F$ is right complementable.

Next we state two propositions:

(7) Let $G, F$ be non empty additive loop structures. Then

(i) for every set $x$ holds $x$ is a point of $G \times F$ iff there exists a point $x_{1}$ of $G$ and there exists a point $x_{2}$ of $F$ such that $x=\left\langle x_{1}, x_{2}\right\rangle$,

(ii) for all points $x, y$ of $G \times F$ and for all points $x_{1}, y_{1}$ of $G$ and for all points $x_{2}, y_{2}$ of $F$ such that $x=\left\langle x_{1}, x_{2}\right\rangle$ and $y=\left\langle y_{1}, y_{2}\right\rangle$ holds $x+y=\left\langle x_{1}+y_{1}\right.$, $\left.x_{2}+y_{2}\right\rangle$, and

(iii) $0_{G \times F}=\left\langle 0_{G}, 0_{F}\right\rangle$.

(8) Let $G, F$ be add-associative right zeroed right complementable non empty additive loop structures, $x$ be a point of $G \times F, x_{1}$ be a point of $G$, and $x_{2}$ be a point of $F$. If $x=\left\langle x_{1}, x_{2}\right\rangle$, then $-x=\left\langle-x_{1},-x_{2}\right\rangle$.

Let $G, F$ be Abelian add-associative right zeroed right complementable strict non empty additive loop structures. One can check that $G \times F$ is strict, Abelian, add-associative, right zeroed, and right complementable.

Let $G, F$ be non empty RLS structures. The functor $G \times F$ yields a strict non empty RLS structure and is defined by:

(Def. 5) $G \times F=\langle($ the carrier of $G) \times($ the carrier of $F)$, prodzero $(G, F)$, $\operatorname{prodadd}(G, F)$, $\operatorname{prodmlt}(G, F)\rangle$.

Let $G, F$ be Abelian non empty RLS structures. Observe that $G \times F$ is Abelian.

Let $G, F$ be add-associative non empty RLS structures. Note that $G \times F$ is add-associative.

Let $G, F$ be right zeroed non empty RLS structures. Note that $G \times F$ is right zeroed.

Let $G, F$ be right complementable non empty RLS structures. One can check that $G \times F$ is right complementable.

Next we state two propositions:

(9) Let $G, F$ be non empty RLS structures. Then

(i) for every set $x$ holds $x$ is a point of $G \times F$ iff there exists a point $x_{1}$ of $G$ and there exists a point $x_{2}$ of $F$ such that $x=\left\langle x_{1}, x_{2}\right\rangle$,

(ii) for all points $x, y$ of $G \times F$ and for all points $x_{1}, y_{1}$ of $G$ and for all points $x_{2}, y_{2}$ of $F$ such that $x=\left\langle x_{1}, x_{2}\right\rangle$ and $y=\left\langle y_{1}, y_{2}\right\rangle$ holds $x+y=\left\langle x_{1}+y_{1}\right.$, $\left.x_{2}+y_{2}\right\rangle$,

(iii) $0_{G \times F}=\left\langle 0_{G}, 0_{F}\right\rangle$, and 
(iv) for every point $x$ of $G \times F$ and for every point $x_{1}$ of $G$ and for every point $x_{2}$ of $F$ and for every real number $a$ such that $x=\left\langle x_{1}, x_{2}\right\rangle$ holds $a \cdot x=\left\langle a \cdot x_{1}, a \cdot x_{2}\right\rangle$.

(10) Let $G, F$ be add-associative right zeroed right complementable non empty RLS structures, $x$ be a point of $G \times F, x_{1}$ be a point of $G$, and $x_{2}$ be a point of $F$. If $x=\left\langle x_{1}, x_{2}\right\rangle$, then $-x=\left\langle-x_{1},-x_{2}\right\rangle$.

Let $G, F$ be vector distributive non empty RLS structures. Note that $G \times$ $F$ is vector distributive.

Let $G, F$ be scalar distributive non empty RLS structures. Note that $G \times F$ is scalar distributive.

Let $G, F$ be scalar associative non empty RLS structures. Observe that $G \times$ $F$ is scalar associative.

Let $G, F$ be scalar unital non empty RLS structures. One can verify that $G \times F$ is scalar unital.

Let $G$ be an Abelian add-associative right zeroed right complementable scalar distributive vector distributive scalar associative scalar unital non empty RLS structure. Note that $\langle G\rangle$ is real-linear-space-yielding.

Let $G, F$ be Abelian add-associative right zeroed right complementable scalar distributive vector distributive scalar associative scalar unital non empty RLS structures. Note that $\langle G, F\rangle$ is real-linear-space-yielding.

\section{Cartesian Products of Real Linear Spaces}

One can prove the following proposition

(11) Let $X$ be a real linear space. Then there exists a function $I$ from $X$ into $\Pi\langle X\rangle$ such that

(i) $I$ is one-to-one and onto,

(ii) for every point $x$ of $X$ holds $I(x)=\langle x\rangle$,

(iii) for all points $v, w$ of $X$ holds $I(v+w)=I(v)+I(w)$,

(iv) for every point $v$ of $X$ and for every element $r$ of $\mathbb{R}$ holds $I(r \cdot v)=r \cdot I(v)$, and

(v) $\quad I\left(0_{X}\right)=0 \prod\langle X\rangle \cdot$

Let $G, F$ be non empty real-linear-space-yielding finite sequences. Observe that $G \frown F$ is real-linear-space-yielding.

We now state three propositions:

(12) Let $X, Y$ be real linear spaces. Then there exists a function $I$ from $X \times$ $Y$ into $\prod\langle X, Y\rangle$ such that

(i) $I$ is one-to-one and onto,

(ii) for every point $x$ of $X$ and for every point $y$ of $Y$ holds $I(x, y)=\langle x$, $y\rangle$,

(iii) for all points $v, w$ of $X \times Y$ holds $I(v+w)=I(v)+I(w)$, 
(iv) for every point $v$ of $X \times Y$ and for every element $r$ of $\mathbb{R}$ holds $I(r \cdot v)=$ $r \cdot I(v)$, and

(v) $\quad I\left(0_{X \times Y}\right)=0 \prod\langle X, Y\rangle$.

(13) Let $X, Y$ be non empty real linear space-sequences. Then there exists a function $I$ from $\prod X \times \prod Y$ into $\prod\left(X^{\frown} Y\right)$ such that

(i) $I$ is one-to-one and onto,

(ii) for every point $x$ of $\prod X$ and for every point $y$ of $\prod Y$ there exist finite sequences $x_{1}, y_{1}$ such that $x=x_{1}$ and $y=y_{1}$ and $I(x, y)=x_{1} \frown y_{1}$,

(iii) for all points $v, w$ of $\prod X \times \prod Y$ holds $I(v+w)=I(v)+I(w)$,

(iv) for every point $v$ of $\prod X \times \prod Y$ and for every element $r$ of $\mathbb{R}$ holds $I(r \cdot v)=r \cdot I(v)$, and

(v) $\quad I\left(0 \prod X \times \prod Y\right)=0 \prod(X-Y)$.

(14) Let $G, F$ be real linear spaces. Then

(i) for every set $x$ holds $x$ is a point of $\prod\langle G, F\rangle$ iff there exists a point $x_{1}$ of $G$ and there exists a point $x_{2}$ of $F$ such that $x=\left\langle x_{1}, x_{2}\right\rangle$,

(ii) for all points $x, y$ of $\prod\langle G, F\rangle$ and for all points $x_{1}, y_{1}$ of $G$ and for all points $x_{2}, y_{2}$ of $F$ such that $x=\left\langle x_{1}, x_{2}\right\rangle$ and $y=\left\langle y_{1}, y_{2}\right\rangle$ holds $x+y=$ $\left\langle x_{1}+y_{1}, x_{2}+y_{2}\right\rangle$,

(iii) ${ }^{0} \prod\langle G, F\rangle=\left\langle 0_{G}, 0_{F}\right\rangle$,

(iv) for every point $x$ of $\prod\langle G, F\rangle$ and for every point $x_{1}$ of $G$ and for every point $x_{2}$ of $F$ such that $x=\left\langle x_{1}, x_{2}\right\rangle$ holds $-x=\left\langle-x_{1},-x_{2}\right\rangle$, and

(v) for every point $x$ of $\prod\langle G, F\rangle$ and for every point $x_{1}$ of $G$ and for every point $x_{2}$ of $F$ and for every real number $a$ such that $x=\left\langle x_{1}, x_{2}\right\rangle$ holds $a \cdot x=\left\langle a \cdot x_{1}, a \cdot x_{2}\right\rangle$.

\section{Cartesian Products of Real Normed Linear Spaces}

Let $G, F$ be non empty normed structures. The functor prodnorm $(G, F)$ yields a function from (the carrier of $G) \times($ the carrier of $F$ ) into $\mathbb{R}$ and is defined by:

(Def. 6) For every point $g$ of $G$ and for every point $f$ of $F$ there exists an element $v$ of $\mathcal{R}^{2}$ such that $v=\langle\|g\|,\|f\|\rangle$ and $(\operatorname{prodnorm}(G, F))(g, f)=|v|$.

Let $G, F$ be non empty normed structures. The functor $G \times F$ yielding a strict non empty normed structure is defined as follows:

(Def. 7) $G \times F=\langle($ the carrier of $G) \times($ the carrier of $F)$, prodzero $(G, F)$, $\operatorname{prodadd}(G, F), \operatorname{prodmlt}(G, F)$, prodnorm $(G, F)\rangle$.

Let $G, F$ be real normed spaces. Observe that $G \times F$ is reflexive, discernible, and real normed space-like.

Let $G, F$ be reflexive discernible real normed space-like scalar distributive vector distributive scalar associative scalar unital Abelian add-associative right 
zeroed right complementable non empty normed structures. One can verify that $G \times F$ is strict, reflexive, discernible, real normed space-like, scalar distributive, vector distributive, scalar associative, scalar unital, Abelian, add-associative, right zeroed, and right complementable.

Let $G$ be a reflexive discernible real normed space-like scalar distributive vector distributive scalar associative scalar unital Abelian add-associative right zeroed right complementable non empty normed structure. One can verify that $\langle G\rangle$ is real-norm-space-yielding.

Let $G, F$ be reflexive discernible real normed space-like scalar distributive vector distributive scalar associative scalar unital Abelian add-associative right zeroed right complementable non empty normed structures. Observe that $\langle G$, $F\rangle$ is real-norm-space-yielding.

One can prove the following propositions:

(15) Let $X, Y$ be real normed spaces. Then there exists a function $I$ from $X \times Y$ into $\prod\langle X, Y\rangle$ such that

(i) $I$ is one-to-one and onto,

(ii) for every point $x$ of $X$ and for every point $y$ of $Y$ holds $I(x, y)=\langle x$, $y\rangle$,

(iii) for all points $v, w$ of $X \times Y$ holds $I(v+w)=I(v)+I(w)$,

(iv) for every point $v$ of $X \times Y$ and for every element $r$ of $\mathbb{R}$ holds $I(r \cdot v)=$ $r \cdot I(v)$,

(v) $0_{\prod\langle X, Y\rangle}=I\left(0_{X \times Y}\right)$, and

(vi) for every point $v$ of $X \times Y$ holds $\|I(v)\|=\|v\|$.

(16) Let $X$ be a real normed space. Then there exists a function $I$ from $X$ into $\prod\langle X\rangle$ such that

(i) $I$ is one-to-one and onto,

(ii) for every point $x$ of $X$ holds $I(x)=\langle x\rangle$,

(iii) for all points $v, w$ of $X$ holds $I(v+w)=I(v)+I(w)$,

(iv) for every point $v$ of $X$ and for every element $r$ of $\mathbb{R}$ holds $I(r \cdot v)=r \cdot I(v)$,

(v) ${ }^{0} \prod\langle X\rangle=I\left(0_{X}\right)$, and

(vi) for every point $v$ of $X$ holds $\|I(v)\|=\|v\|$.

Let $G, F$ be non empty real-norm-space-yielding finite sequences. One can check that $G^{\frown} F$ is non empty and real-norm-space-yielding.

One can prove the following propositions:

(17) Let $X, Y$ be non empty real norm space-sequences. Then there exists a function $I$ from $\prod X \times \prod Y$ into $\prod\left(X^{\frown} Y\right)$ such that

(i) $I$ is one-to-one and onto,

(ii) for every point $x$ of $\prod X$ and for every point $y$ of $\prod Y$ there exist finite sequences $x_{1}, y_{1}$ such that $x=x_{1}$ and $y=y_{1}$ and $I(x, y)=x_{1} \frown y_{1}$,

(iii) for all points $v, w$ of $\prod X \times \prod Y$ holds $I(v+w)=I(v)+I(w)$, 
(iv) for every point $v$ of $\prod X \times \prod Y$ and for every element $r$ of $\mathbb{R}$ holds $I(r \cdot v)=r \cdot I(v)$,

(v) $\quad I\left(0 \prod X \times \prod Y\right)=0 \prod(X \frown Y)$, and

(vi) for every point $v$ of $\prod X \times \prod Y$ holds $\|I(v)\|=\|v\|$.

(18) Let $G, F$ be real normed spaces. Then

(i) for every set $x$ holds $x$ is a point of $G \times F$ iff there exists a point $x_{1}$ of $G$ and there exists a point $x_{2}$ of $F$ such that $x=\left\langle x_{1}, x_{2}\right\rangle$,

(ii) for all points $x, y$ of $G \times F$ and for all points $x_{1}, y_{1}$ of $G$ and for all points $x_{2}, y_{2}$ of $F$ such that $x=\left\langle x_{1}, x_{2}\right\rangle$ and $y=\left\langle y_{1}, y_{2}\right\rangle$ holds $x+y=\left\langle x_{1}+y_{1}\right.$, $\left.x_{2}+y_{2}\right\rangle$,

(iii) $0_{G \times F}=\left\langle 0_{G}, 0_{F}\right\rangle$,

(iv) for every point $x$ of $G \times F$ and for every point $x_{1}$ of $G$ and for every point $x_{2}$ of $F$ such that $x=\left\langle x_{1}, x_{2}\right\rangle$ holds $-x=\left\langle-x_{1},-x_{2}\right\rangle$,

(v) for every point $x$ of $G \times F$ and for every point $x_{1}$ of $G$ and for every point $x_{2}$ of $F$ and for every real number $a$ such that $x=\left\langle x_{1}, x_{2}\right\rangle$ holds $a \cdot x=\left\langle a \cdot x_{1}, a \cdot x_{2}\right\rangle$, and

(vi) for every point $x$ of $G \times F$ and for every point $x_{1}$ of $G$ and for every point $x_{2}$ of $F$ such that $x=\left\langle x_{1}, x_{2}\right\rangle$ there exists an element $w$ of $\mathcal{R}^{2}$ such that $w=\left\langle\left\|x_{1}\right\|,\left\|x_{2}\right\|\right\rangle$ and $\|x\|=|w|$.

(19) Let $G, F$ be real normed spaces. Then

(i) for every set $x$ holds $x$ is a point of $\prod\langle G, F\rangle$ iff there exists a point $x_{1}$ of $G$ and there exists a point $x_{2}$ of $F$ such that $x=\left\langle x_{1}, x_{2}\right\rangle$,

(ii) for all points $x, y$ of $\prod\langle G, F\rangle$ and for all points $x_{1}, y_{1}$ of $G$ and for all points $x_{2}, y_{2}$ of $F$ such that $x=\left\langle x_{1}, x_{2}\right\rangle$ and $y=\left\langle y_{1}, y_{2}\right\rangle$ holds $x+y=$ $\left\langle x_{1}+y_{1}, x_{2}+y_{2}\right\rangle$,

(iii) ${ }^{0} \prod_{\langle G, F\rangle}=\left\langle 0_{G}, 0_{F}\right\rangle$,

(iv) for every point $x$ of $\prod\langle G, F\rangle$ and for every point $x_{1}$ of $G$ and for every point $x_{2}$ of $F$ such that $x=\left\langle x_{1}, x_{2}\right\rangle$ holds $-x=\left\langle-x_{1},-x_{2}\right\rangle$,

(v) for every point $x$ of $\prod\langle G, F\rangle$ and for every point $x_{1}$ of $G$ and for every point $x_{2}$ of $F$ and for every real number $a$ such that $x=\left\langle x_{1}, x_{2}\right\rangle$ holds $a \cdot x=\left\langle a \cdot x_{1}, a \cdot x_{2}\right\rangle$, and

(vi) for every point $x$ of $\prod\langle G, F\rangle$ and for every point $x_{1}$ of $G$ and for every point $x_{2}$ of $F$ such that $x=\left\langle x_{1}, x_{2}\right\rangle$ there exists an element $w$ of $\mathcal{R}^{2}$ such that $w=\left\langle\left\|x_{1}\right\|,\left\|x_{2}\right\|\right\rangle$ and $\|x\|=|w|$.

Let $X, Y$ be complete real normed spaces. Observe that $X \times Y$ is complete.

We now state several propositions:

(20) Let $X, Y$ be non empty real norm space-sequences. Then there exists a function $I$ from $\prod\left\langle\prod X, \prod Y\right\rangle$ into $\prod\left(X^{\frown} Y\right)$ such that

(i) $I$ is one-to-one and onto,

(ii) for every point $x$ of $\prod X$ and for every point $y$ of $\prod Y$ there exist finite sequences $x_{1}, y_{1}$ such that $x=x_{1}$ and $y=y_{1}$ and $I(\langle x, y\rangle)=x_{1} \frown y_{1}$, 
(iii) for all points $v, w$ of $\prod\left\langle\prod X, \prod Y\right\rangle$ holds $I(v+w)=I(v)+I(w)$,

(iv) for every point $v$ of $\prod\left\langle\prod X, \Pi Y\right\rangle$ and for every element $r$ of $\mathbb{R}$ holds $I(r \cdot v)=r \cdot I(v)$,

(v) $\quad I\left(0 \prod\left\langle\prod X, \prod Y\right\rangle\right)=0 \prod(X \wedge Y)$, and

(vi) for every point $v$ of $\prod\left\langle\prod X, \prod Y\right\rangle$ holds $\|I(v)\|=\|v\|$.

(21) Let $X, Y$ be non empty real linear spaces. Then there exists a function $I$ from $X \times Y$ into $X \times \prod\langle Y\rangle$ such that

(i) $I$ is one-to-one and onto,

(ii) for every point $x$ of $X$ and for every point $y$ of $Y$ holds $I(x, y)=\langle x$, $\langle y\rangle\rangle$,

(iii) for all points $v, w$ of $X \times Y$ holds $I(v+w)=I(v)+I(w)$,

(iv) for every point $v$ of $X \times Y$ and for every element $r$ of $\mathbb{R}$ holds $I(r \cdot v)=$ $r \cdot I(v)$, and

(v) $\quad I\left(0_{X \times Y}\right)=0_{X \times \prod\langle Y\rangle}$.

(22) Let $X$ be a non empty real linear space-sequence and $Y$ be a real linear space. Then there exists a function $I$ from $\prod X \times Y$ into $\prod\left(X^{\frown}\langle Y\rangle\right)$ such that

(i) $I$ is one-to-one and onto,

(ii) for every point $x$ of $\prod X$ and for every point $y$ of $Y$ there exist finite sequences $x_{1}, y_{1}$ such that $x=x_{1}$ and $\langle y\rangle=y_{1}$ and $I(x, y)=x_{1}{ }^{\curvearrowleft} y_{1}$,

(iii) for all points $v, w$ of $\prod X \times Y$ holds $I(v+w)=I(v)+I(w)$,

(iv) for every point $v$ of $\prod X \times Y$ and for every element $r$ of $\mathbb{R}$ holds $I(r \cdot v)=r \cdot I(v)$, and

(v) $\quad I\left(0 \prod_{X \times Y}\right)=0 \prod_{(X \sim\langle Y\rangle)}$.

(23) Let $X, Y$ be non empty real normed spaces. Then there exists a function $I$ from $X \times Y$ into $X \times \prod\langle Y\rangle$ such that

(i) $I$ is one-to-one and onto,

(ii) for every point $x$ of $X$ and for every point $y$ of $Y$ holds $I(x, y)=\langle x$, $\langle y\rangle\rangle$,

(iii) for all points $v, w$ of $X \times Y$ holds $I(v+w)=I(v)+I(w)$,

(iv) for every point $v$ of $X \times Y$ and for every element $r$ of $\mathbb{R}$ holds $I(r \cdot v)=$ $r \cdot I(v)$,

(v) $I\left(0_{X \times Y}\right)=0_{X \times \prod\langle Y\rangle}$, and

(vi) for every point $v$ of $X \times Y$ holds $\|I(v)\|=\|v\|$.

(24) Let $X$ be a non empty real norm space-sequence and $Y$ be a real normed space. Then there exists a function $I$ from $\prod X \times Y$ into $\prod\left(X^{\frown}\langle Y\rangle\right)$ such that

(i) $I$ is one-to-one and onto,

(ii) for every point $x$ of $\prod X$ and for every point $y$ of $Y$ there exist finite sequences $x_{1}, y_{1}$ such that $x=x_{1}$ and $\langle y\rangle=y_{1}$ and $I(x, y)=x_{1} \frown y_{1}$,

(iii) for all points $v, w$ of $\prod X \times Y$ holds $I(v+w)=I(v)+I(w)$, 
(iv) for every point $v$ of $\prod X \times Y$ and for every element $r$ of $\mathbb{R}$ holds $I(r \cdot v)=r \cdot I(v)$,

(v) $\quad I\left(0_{X \times Y}\right)=0 \prod_{(X \frown\langle Y\rangle)}$, and

(vi) for every point $v$ of $\prod X \times Y$ holds $\|I(v)\|=\|v\|$.

\section{REFERENCES}

[1] Grzegorz Bancerek. König's theorem. Formalized Mathematics, 1(3):589-593, 1990.

2] Grzegorz Bancerek. The ordinal numbers. Formalized Mathematics, 1(1):91-96, 1990.

[3] Grzegorz Bancerek and Krzysztof Hryniewiecki. Segments of natural numbers and finite sequences. Formalized Mathematics, 1(1):107-114, 1990.

[4] Nicolas Bourbaki. Topological vector spaces: Chapters 1-5. Springer, 1981.

[5] Czesław Byliński. Binary operations. Formalized Mathematics, 1(1):175-180, 1990.

[6] Czesław Byliński. Finite sequences and tuples of elements of a non-empty sets. Formalized Mathematics, 1(3):529-536, 1990.

[7] Czesław Byliński. Functions and their basic properties. Formalized Mathematics, 1(1):5565, 1990.

[8] Czesław Byliński. Functions from a set to a set. Formalized Mathematics, 1(1):153-164, 1990

[9] Czesław Byliński. Some basic properties of sets. Formalized Mathematics, 1(1):47-53, 1990.

[10] Czesław Byliński. The sum and product of finite sequences of real numbers. Formalized Mathematics, 1(4):661-668, 1990.

[11] Agata Darmochwal. The Euclidean space. Formalized Mathematics, 2(4):599-603, 1991.

[12] Noboru Endou, Yasunari Shidama, and Keiichi Miyajima. The product space of real normed spaces and its properties. Formalized Mathematics, 15(3):81-85, 2007, doi:10.2478/v10037-007-0010-y.

[13] Beata Padlewska and Agata Darmochwał. Topological spaces and continuous functions. Formalized Mathematics, 1(1):223-230, 1990.

[14] Jan Popiołek. Real normed space. Formalized Mathematics, 2(1):111-115, 1991.

[15] Yasunari Shidama. Banach space of bounded linear operators. Formalized Mathematics, 12(1):39-48, 2004.

[16] Andrzej Trybulec. Domains and their Cartesian products. Formalized Mathematics, $1(\mathbf{1}): 115-122,1990$

[17] Wojciech A. Trybulec. Pigeon hole principle. Formalized Mathematics, 1(3):575-579, 1990.

[18] Wojciech A. Trybulec. Vectors in real linear space. Formalized Mathematics, 1(2):291-296, 1990.

[19] Zinaida Trybulec. Properties of subsets. Formalized Mathematics, 1(1):67-71, 1990.

[20] Edmund Woronowicz. Relations and their basic properties. Formalized Mathematics, 1(1):73-83, 1990. 